\title{
Toroidal Plasma Rotation Induced by the Dynamic Ergodic Divertor in the TEXTOR Tokamak
}

K. H. Finken, ${ }^{1}$ S. S. Abdullaev, ${ }^{1}$ M. F. M. de Bock, ${ }^{2}$ M. von Hellermann, ${ }^{2}$ M. Jakubowski, ${ }^{1}$ R. Jaspers, ${ }^{2}$ H. R. Koslowski, ${ }^{1}$ A. Krämer-Flecken, ${ }^{1}$ M. Lehnen, ${ }^{1}$ Y. Liang, ${ }^{1}$ A. Nicolai, ${ }^{1}$ R. C. Wolf, ${ }^{1}$ O. Zimmermann, ${ }^{1}$ M. de Baar, ${ }^{2}$ G. Bertschinger, ${ }^{1}$ W. Biel, ${ }^{1}$ S. Brezinsek, ${ }^{1}$ C. Busch, ${ }^{1}$ A. J. H. Donné, ${ }^{2}$ H. G. Esser, ${ }^{1}$ E. Farshi, ${ }^{2}$ H. Gerhauser, ${ }^{1}$ B. Giesen, ${ }^{1}$ D. Harting, ${ }^{1}$ J. A. Hoekzema, ${ }^{1}$ G. M. D. Hogeweij,${ }^{2}$ P.W. Hüttemann, ${ }^{1}$ S. Jachmich, ${ }^{3}$ K. Jakubowska, ${ }^{2}$ D. Kalupin, ${ }^{1}$ F. Kelly, ${ }^{1}$ Y. Kikuchi, ${ }^{8}$ A. Kirschner, ${ }^{1}$ R. Koch,${ }^{3}$ M. Korten, ${ }^{1}$ A. Kreter, ${ }^{1}$ J. Krom, ${ }^{1}$ U. Kruezi, ${ }^{1}$ A. Lazaros, ${ }^{2}$ A. Litnovsky, ${ }^{1}$ X. Loozen, ${ }^{1}$ N. J. Lopes Cardozo, ${ }^{2}$ A. Lyssoivan, ${ }^{3}$ O. Marchuk, ${ }^{1}$ G. Matsunaga,${ }^{1}$ Ph. Mertens, ${ }^{1}$ A. Messiaen,${ }^{3}$ O. Neubauer, ${ }_{1}^{1}$ N. Noda,${ }^{4}$ V. Philipps, ${ }^{1}$ A. Pospieszczyk, ${ }^{1}$ D. Reiser, ${ }^{1}$ D. Reiter, ${ }^{1}$ A. L. Rogister, ${ }^{1}$ M. Sakamoto, ${ }^{7}$ A. Savtchkov, ${ }^{1}$ U. Samm, ${ }^{1}$ O. Schmitz, ${ }^{1}$ R. P. Schorn, ${ }^{1}$ B. Schweer, ${ }^{1}$ F. C. Schüller, ${ }^{2}$ G. Sergienko, ${ }^{1}$ K. H. Spatschek, ${ }^{5}$ G. Telesca, ${ }^{6}$ M. Tokar, ${ }^{1}$ R. Uhlemann, ${ }^{1}$ B. Unterberg, ${ }^{1}$ G. Van Oost, ${ }^{6}$ T. Van Rompuy, ${ }^{6}$ G. Van Wassenhove,${ }^{3}$ E. Westerhof, ${ }^{2}$ R. Weynants, ${ }^{3}$ S. Wiesen, ${ }^{1}$ and Y. H. Xu ${ }^{3}$

${ }^{1}$ Trilateral Euregio Cluster: Institut für Plasmaphysik, Forschungszentrum Jülich, EURATOM Association, D-52425 Jülich, Germany

${ }^{2}$ FOM Institute for Plasma Physics Rijnhuizen, EURATOM Association, Postbus 1207, NL-3430 BE Nieuwegein, The Netherlands ${ }^{3}$ Laboratoire de Physique des Plasmas/Laboratorium voor Plasmafysica, ERM/KMS, EURATOM Association, B-1000 Brussels, Belgium

${ }^{4}$ National Institute for Fusion Science, Toki, Japan

${ }^{5}$ Institut für Theoretische Physik, Heinrich-Heine Universität Düsseldorf, D-40225 Düsseldorf, Germany

${ }^{6}$ Department of Applied Physics, Ghent University, Rozier 44, B-9000 Ghent, Belgium

${ }^{7}$ Research Institute for Applied Mechanics, Kyushu University, Fukuoka, Japan

${ }^{8}$ Nagoya University, Nagoya, Japan

(Received 28 July 2004; published 7 January 2005)

The first results of the Dynamic Ergodic Divertor in TEXTOR, when operating in the $m / n=3 / 1$ mode configuration, are presented. The deeply penetrating external magnetic field perturbation of this configuration increases the toroidal plasma rotation. Staying below the excitation threshold for the $m / n=2 / 1$ tearing mode, this toroidal rotation is always in the direction of the plasma current, even if the toroidal projection of the rotating magnetic field perturbation is in the opposite direction. The observed toroidal rotation direction is consistent with a radial electric field, generated by an enhanced electron transport in the ergodic layers near the resonances of the perturbation. This is an effect different from theoretical predictions, which assume a direct coupling between rotating perturbation and plasma to be the dominant effect of momentum transfer.

PACS numbers: 52.55.Fa, 52.30.-q, 52.35.Vd, 52.55.Tn

Helical magnetic field perturbations are introduced in tokamak plasmas to study, on the one hand, the ergodic divertor concept $[1,2]$ and, on the other hand, the interaction of such perturbations with the magnetohydrodynamics (MHD) stability of the plasma [3,4]. Recent experiments, for instance, suggest a control method to mitigate edge localized modes while maintaining the pedestal pressure and thus plasma confinement [5-7]. However, open questions remain, in particular, with regard to the influence on the momentum transport of the plasma. Indeed, one motivation to equip the tokamak TEXTOR with the Dynamic Ergodic Divertor (DED) [8] was to be able to study the interaction between helical magnetic field perturbations and plasma transport and stability.

The DED consists of 16 magnetic perturbation coils (four quadruples), plus two additional coils for the compensation of the magnetic field imperfections at the feeder regions of the coils. The coils wind helically around the inner side of the torus (major radius: $R=$
$1.75 \mathrm{~m}$; minor radius of the circular plasma cross section typically $a=0.47 \mathrm{~m}$ ) with a pitch corresponding to the magnetic field lines of the magnetic flux surface with a safety factor of $q=3$. Depending on the choice of coil connections to the power supplies, base modes with different poloidal and toroidal mode numbers can be produced. For the DED these are $m / n=12 / 4,6 / 2$, and $3 / 1$. The penetration depth into the plasma strongly depends on the mode numbers: While the $m / n=12 / 4$ affects the edge plasma only, the $m / n=3 / 1$ mode reaches into the plasma center (the maximum radial magnetic field component achievable by the DED at the $q=2$ surface is $\sim 10^{-3}$ of the total magnetic field).

In this Letter we present results obtained by the $m / n=$ $3 / 1$ mode operation. Covering about one-third of the poloidal cross section of the torus, the mode spectrum of the DED does not contain many sidebands. For the $m / n=3 / 1$ configuration the three dominant resonant components inside the plasma are $m=1,2$, and 3. In Fig. 1 their strengths at the respective resonances are 

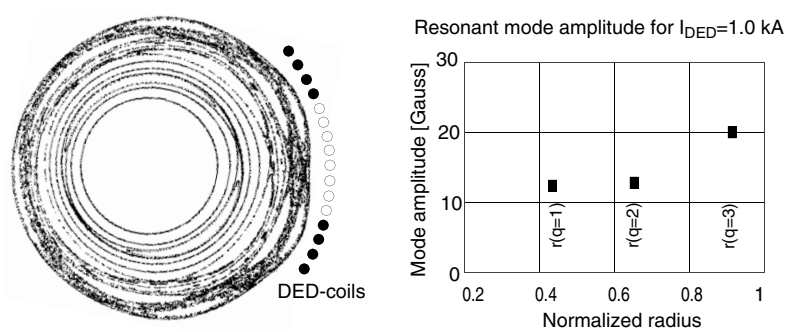

FIG. 1. Poincaré plot of the magnetic field topology with (static) DED for the $m / n=3 / 1$ configuration, calculated from a superposition of the perturbation field (for a DED current, $I_{\mathrm{DED}}=1.0 \mathrm{kA}$ ) and a plasma equilibrium with an edge safety factor of $q(a)=4.5$. On the right the underlying amplitudes of the magnetic field perturbation for the $m=1,2$, and 3 components are plotted at their resonance positions.

exemplified. Also illustrated in the form of a Poincaré plot is the corresponding magnetic field topology, calculated from a linear superposition of the (static) magnetic field perturbation by the DED and a plasma equilibrium [8-11]. The magnetic island structures and the ergodic regions in their vicinity can be clearly seen.

The unique feature of the DED is the possibility to rotate the magnetic fields with frequencies of up to $10 \mathrm{kHz}$ by supplying the coils with ac currents. This frequency is of the order of the diamagnetic velocity at the plasma edge region or of the central toroidal rotation velocity achievable by about $1 \mathrm{MW}$ of unidirectional neutral beam injection. To our knowledge the only other perturbation field experiments with similar properties are the much smaller research tokamak CSTN at Nagoya University [12] and the tokamak TEXT [13], which however was limited to a much lower perturbation level.

The course of the experiments, presented here, can be outlined as follows (Fig. 2). After discharge initiation, first neutral beam injection (NBI) is applied. NBI is required for the measurement of the toroidal plasma velocity by charge exchange recombination spectroscopy (CXRS) and, depending on the applied beam lines and the level of injected power, constitutes a significant source of toroidal angular momentum. TEXTOR is equipped with two beam lines which inject toroidally either in (co-NBI) or opposite to the direction of the plasma current (counter-NBI). Once the plasma has reached stationary conditions with respect to plasma current, heating power, electron and ion temperatures, and electron density, the currents in the DED coils are slowly ramped up $(\approx 0.7 \mathrm{kA} / \mathrm{s})$ to a preprogrammed level. This level is held constant for about half a second, after which the DED is switched off again.

The influence on the toroidal plasma rotation has been studied for different combinations of perturbation field rotation direction and frequency: static perturbation without rotation, $1 \mathrm{kHz}$ corotation in the direction of the plasma current, and $1 \mathrm{kHz}$ counterrotation opposite

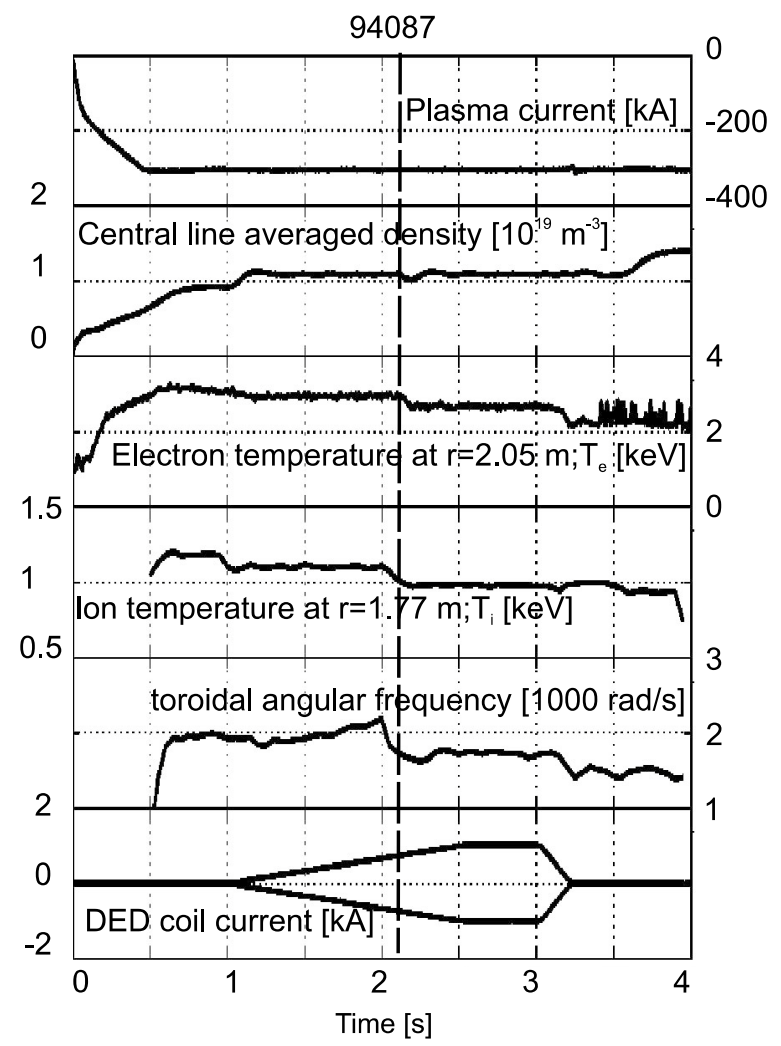

FIG. 2. Characteristic time traces of discharge parameters for a case where static DED fields have been applied. Shown are (from top to bottom) plasma current, central line averaged density, electron and ion temperature, central toroidal angular frequency, and currents of two independent DED coils. The vertical line indicates the onset of a tearing mode, induced by the DED. The time traces of ion temperature and rotation frequency also indicate the time of NBI $(0.3 \mathrm{MW})$. At the shown radii temperatures and toroidal angular frequency drop sharply at the onset of the tearing mode.

to the plasma current. NBI has been varied between 0.3 MW coinjection, 1.5 MW balanced injection (coinjected and counterinjected powers balance each other), and $1.5 \mathrm{MW}$ coinjection. On TEXTOR the NBI power is varied by inserting a $V$-shaped target with a reduced opening into the beam line, thereby reducing the power injected into the plasma. At low power $(0.3 \mathrm{MW})$ the auxiliary heating is similar to the Ohmic heating by $300 \mathrm{kA}$ of plasma current. Since the DED perturbation field is helical, it has poloidal and toroidal components. Likewise, in the dynamic case, the field rotation is in poloidal and toroidal directions (with a ratio of toroidal to poloidal components of only $10 \%$ ). For rotating DED fields this means that, if the toroidal projection of the rotation is in the cocurrent direction, the poloidal projection is in the ion diamagnetic drift direction.

The time evolution of characteristic parameters of a discharge with static DED and low NBI power is shown in Fig. 2. During the application of the magnetic field per- 
turbation two phases can be distinguished. First, the DED coil currents rise without a major impact on the plasma behavior. Plasma temperatures, density, and energy do not change significantly. The only remarkable change is a slight increase of the angular frequency with an increasing DED current of about $20 \%$ with regard to the rotation produced by NBI alone.

The second phase is characterized by the formation of a $m / n=2 / 1$ MHD instability with a saturated mode amplitude, once the current per DED coil exceeds a critical value of about $0.8 \mathrm{kA}$, which corresponds to a magnetic field perturbation of $1.0 \times 10^{-3} \mathrm{~T}$ at the $q=2$ surface. Local flattening of temperature and density profiles in the vicinity of the $q=2$ surface suggest the formation of a tearing mode. The helicity is deduced from the relative phase evolution of Mirnov coil signals, which are taken at different poloidal and toroidal positions. Once excited, the mode locks to the external magnetic field perturbation; i.e., for a static DED it does not move with respect to the plasma vessel. After the DED is switched off, the mode persists but starts to rotate with a frequency of $\approx 1.5 \mathrm{kHz}$, which is seen on both the Mirnov coil signals at the plasma boundary and the soft $\mathrm{x}$-ray emission from the plasma core. In some cases, the rotation of the tearing mode does not start immediately after the switch off of the DED but is delayed until also the NBI is turned off. The excitation of the tearing mode is very reproducible and the critical perturbation threshold varies with plasma parameters, such as density, plasma beta, or toroidal plasma rotation [14]. To obtain a stationary saturated mode an edge safety factor $q(a) \geq 4.5$ is required. At lower $q(a)$ the plasma tends to disrupt.

The tearing mode is accompanied by a $12 \%$ loss of thermal energy, calculated from the profiles of electron and ion temperature, and electron density. The electron density shows only a small dip at the onset of the tearing mode, caused by the feedback control of the density. Set to a level of $2 \times 10^{19} \mathrm{~m}^{-3}$, the loss of particle confinement is compensated by a stronger gas fueling which keeps the density constant. The observed dip is a consequence of the finite response time of the feedback control system.

Most noticeable, however, is the change of the central angular toroidal frequency, $\omega_{\phi}=v_{\phi} / R$ (where $v_{\phi}$ is the Doppler velocity obtained from CXRS). Figure 3 shows the temporal profile evolution of ion plasma rotation, ion temperature, and electron temperature. Owing to technical restrictions, the CXRS measurement covers only the inner two-thirds of the plasma, ranging from the center to the $q=2$ surface. The $T_{e}$ measurement is derived from two electron cyclotron emission (ECE) systems which are separated toroidally by $110^{\circ}$. The upper (red) curves in Fig. 3(c) correspond to the case prior to the onset of the mode. The two lower curves represent the $T_{e}$ distribution after the onset of the mode, showing an overall reduction
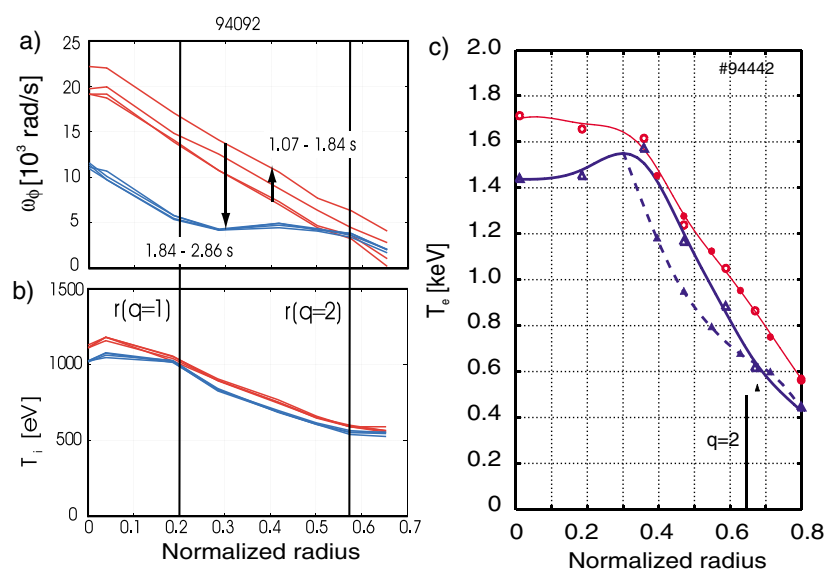

FIG. 3 (color). Temporal evolution of the radial profiles of (a) toroidal rotation, (b) ion temperature, and (c) electron temperature. The increase of the DED current before the onset of the mode is denoted in red; the times after the mode onset are shown in blue. The onset of the mode reduces the central temperature peaking and flattens $\omega_{\phi}$ between the $q=1$ and 2 surfaces. In addition $T_{e}$ can just resolve the local flattening due to the island formation at the $q=2$ surface.

of the ECE temperature, measured close to the X point of the tearing mode (solid line), and a local flattening at the $\mathrm{O}$ point (dashed line). This flattening allows an estimate of the island width of 5-6 cm, which is about twice the value of the vacuum island $(3.2 \mathrm{~cm})$ at the mode onset. During the first phase, when the DED coil currents start increasing, $\omega_{\phi}$ rises over the whole profile, while the ion temperature does not change. At the onset of the tearing mode $\omega_{\phi}$ drops in the plasma center by almost a factor of 2 , in contrast to the vicinity of the $q=2$ surface, where it further increases. This results in a flat rotation profile between the $q=1$ and 2 surfaces, as if in this region the plasma moved like a rigid body. The island width, however, is considerably smaller than the flat part of $v_{\phi}$. Both ion and electron temperature do not show such a pronounced change, which is also reflected in the quite moderate drop of plasma energy.

With rotating DED field the temporal evolution of the experiment is basically the same as the one illustrated in Fig. 2. The only difference is that the DED coils are now supplied with ac currents to produce a rotating magnetic field perturbation. Rotating the DED with a frequency of $1 \mathrm{kHz}$ opposite to the plasma current and NBI (counterrotation), the behavior of the plasma is essentially the same as in the static case: At first, a slight increase of the toroidal plasma rotation with increasing DED current is observed until the threshold for the excitation of the $m / n=2 / 1$ tearing mode is exceeded (Fig. 4). Considering now the effective current (rms) in the DED coils, this threshold is about the same as in the static case. Again, the appearance of the tearing mode coincides with a flattening of the angular frequency pro- 


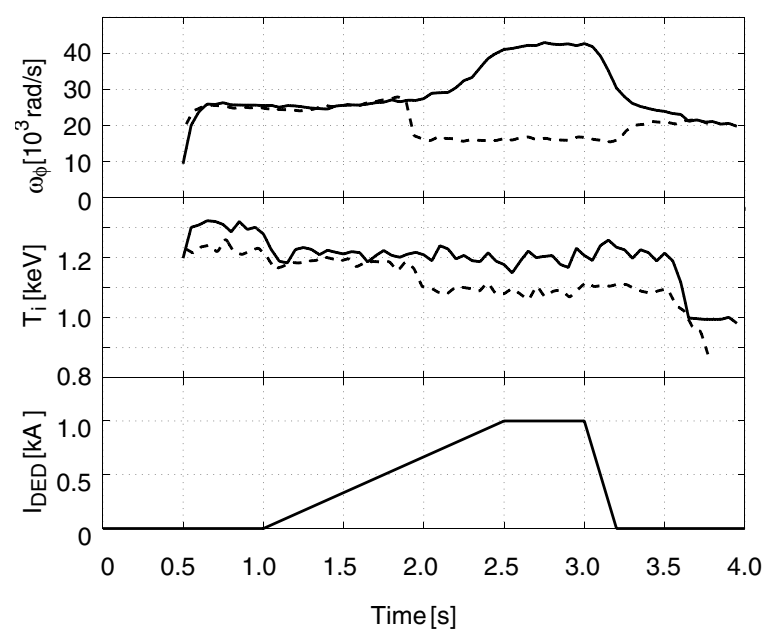

FIG. 4. Temporal evolution of the central toroidal angular frequency and ion temperature for corotating (solid line) and counterrotating (dashed line) DED. The DED current amplitude, which is the same in both cases, is indicated by the trace at the bottom of the graph.

file. Consistent with the observations in the static case (Fig. 3), the core plasma rotation does not completely brake or lock to the mode. Instead a finite corotation is preserved $\left[f=\omega_{\phi} /(2 \pi) \approx 1 \mathrm{kH} z\right]$, while the DED perturbation fields are rotating with about the same frequency in the opposite direction.

The most prominent effect is seen when applying a corotating DED field (with a frequency of $1 \mathrm{kHz}$ ). A tearing mode is not excited, although the effective DED current is clearly above the threshold required to excite a tearing mode in the static or counterrotating cases. Instead, as illustrated in Fig. 4, the toroidal rotation continues to rise with the DED current to almost twice the initial level.

The relation between the change of the central toroidal rotation and DED current amplitude for cases with corotating or counterrotating DED and different NBI powers and injection directions is summarized in Fig. 5. Accordingly, the initial toroidal velocity varies between 0 (balanced NBI) and $80 \mathrm{krad} / \mathrm{s}$ (1.5 MW co-NBI). In all cases, however, $\Delta \omega_{\phi}$ increases with DED current. Only the level at which the tearing mode sets in, and hence the achievable increase of toroidal rotation, is different. Interpreting the strength of the DED current as the degree of ergodization [15], the strong dependence of $\Delta \omega_{\phi}$ on the DED current is supporting evidence that the magnetic field ergodization is the cause for the strong toroidal spinup of the plasma. The fact that the rise of the rotation is independent of the DED rotation direction, as long as the tearing mode is not excited, indicates the presence of an effect different to a direct resonant coupling between DED and plasma [16,17].

A tentative explanation could be an enhanced radial transport of the electrons in the open field lines of the

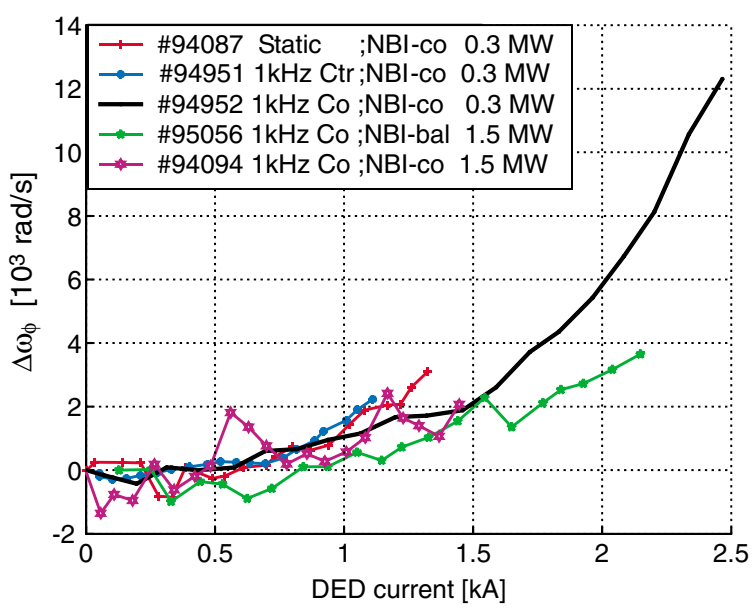

FIG. 5 (color). Dependence of the change of the central toroidal angular frequency, $\Delta \omega_{\phi}$, on DED current, $I_{\mathrm{DED}}$, for discharges with corotating and counterrotating perturbation field and different beam powers and injection directions. While the initial toroidal rotation frequencies vary between 0 (balanced NBI) and $80 \mathrm{krad} / \mathrm{s}$ (1.5 MW co-NBI), all cases show a comparable increase of $\Delta \omega_{\phi}$ with $I_{\mathrm{DED}}$.

ergodic boundary together with the ambipolarity constraint for the ions. This leads to a charge separation which is counteracted by the buildup of a radial electric field, $E_{r}$. The direction of the observed increase of $v_{\phi}$ is consistent with such a mechanism, assuming that the $v_{\phi} \times B_{\theta}$ component in the force balance ( $B_{\theta}$ is the poloidal magnetic field) is the dominant term of the change of $E_{r}$.

In conclusion, a toroidal spin-up of the plasma has been produced by applying the DED in the TEXTOR tokamak. The scaling of the effect with perturbation field amplitude suggests that ergodic layers inside the plasma generate the rotation. At least the observed toroidal rotation direction agrees with a radial electric field, which is produced by an enhanced electron transport in such ergodic layers. The absence of substantial confinement changes suggests that also the plasma viscosity remains unaffected. Even if it changed, the case without net momentum input (balanced NBI) does not support an influence of the viscosity on the increase of rotation. Thus the radial electric field could serve as the required momentum source. Assuming that the toroidal rotation profile smoothly goes to zero at the plasma edge, the measured evolutions of $\omega_{\phi}$ and plasma pressure do not indicate strong changes of the $E \times B$ shearing rate, which would explain why the confinement does not change.

To achieve a significant increase of toroidal plasma rotation the perturbation field amplitude and the corresponding ergodization have to be sufficiently large and, at the same time, the formation of a tearing mode has to be avoided. In the parameter range studied this is accomplished if the DED rotation is parallel to plasma current 
and NBI direction, independent of the NBI power. Why the tearing mode threshold is lower for a corotating DED field has yet to be explained.

[1] T. E. Evans et al., J. Nucl. Mater. 196-198, 421 (1992).

[2] P. Ghendrih et al., Nucl. Fusion 42, 1221 (2002).

[3] T. C. Hender et al., Nucl. Fusion 32, 2091 (1992).

[4] R. Fitzpatrick, Nucl. Fusion 33, 1049 (1993).

[5] H. Tamai et al., in Proceedings of the 15th International Conference on Plasma Physics and Controlled Fusion (IAEA, Seville, 1995), Vol. 1, p. 137.

[6] A. Grosman et al., J. Nucl. Mater. 313-316, 1314 (2003).

[7] T. Evans et al., Phys. Rev. Lett. 92, 235003 (2004).
[8] Special Issue, edited by K. H. Finken, Fusion Eng. Des. 37, 335 (1997).

[9] K. Finken, S. S. Abdullaev, A. Kaleck, and G. H. Wolf, Nucl. Fusion 39, 637 (1999).

[10] M. Kobayashi et al., Contrib. Plasma Phys. 42, 163 (2002).

[11] S. Abdullaev et al., Nucl. Fusion 43, 299 (2003).

[12] M. Kobayashi et al., Nucl. Fusion 40, 181 (2000).

[13] M. Foster, S. McCool, and A. Wooton, Nucl. Fusion 35, 329 (1995).

[14] H. R. Koslowski et al., in Proceedings of the 31st European Physics Society Conference on Plasma Physics, London, 2004 (European Physical Society, Mulhouse, 2004), p. P1.124.

[15] M. C. Jakubowski et al., Nucl. Fusion 44, 395 (2004).

[16] K. H. Finken, Nucl. Fusion 39, 707 (1999).

[17] A. G. Elfimov et al., Nucl. Fusion 44, 83 (2004). 